\title{
Segmentation of medial temporal subregions reveals early right-sided involvement in semantic variant PPA
}

Martina Bocchetta ${ }^{1}$ D, Juan Eugenio Iglesias², Lucy L. Russell', Caroline V. Greaves ${ }^{1}$, Charles R. Marshall', Marzia A. Scelsi ${ }^{2}$, David M. Cash ${ }^{1,2}$, Sebastien Ourselin ${ }^{3}$, Jason D. Warren ${ }^{1}$ and Jonathan D. Rohrer ${ }^{1 *}$

\begin{abstract}
Background: Semantic variant of primary progressive aphasia (SVPPA) is a subtype of frontotemporal dementia characterized by asymmetric temporal atrophy.

Methods: We investigated the pattern of medial temporal lobe atrophy in 24 sVPPA patients compared to 72 controls using novel approaches to segment the hippocampal and amygdalar subregions on MRIs. Based on semantic knowledge scores, we split the svPPA group into 3 subgroups of early, middle and late disease stage.

Results: Early stage: all left amygdalar and hippocampal subregions (except the tail) were affected in svPPA (21-35\% smaller than controls), together with the following amygdalar nuclei in the right hemisphere: lateral, accessory basal and superficial (15-23\%). On the right, only the temporal pole was affected among the cortical regions. Middle stage: the left hippocampal tail became affected (28\%), together with the other amygdalar nuclei (22-26\%), and CA4 (15\%) on the right, with orbitofrontal cortex and subcortical structures involvement on the left, and more posterior temporal lobe on the right. Late stage: the remaining right hippocampal regions (except the tail) (19-24\%) became affected, with more posterior left cortical and right extra-temporal anterior cortical involvement.
\end{abstract}

Conclusions: With advanced subregions segmentation, it is possible to detect early involvement of the right medial temporal lobe in sVPPA that is not detectable by measuring the amygdala or hippocampus as a whole.

Keywords: Semantic variant PPA, Magnetic resonance imaging, Medial temporal subregions

\section{Introduction}

Semantic variant of primary progressive aphasia (svPPA) is a subtype of frontotemporal dementia (FTD), characterized clinically by anomia and impaired single-word comprehension. It is associated with a characteristic pattern of asymmetrical antero-inferior temporal lobe atrophy [1-3]. Previous studies of svPPA have shown early left medial temporal lobe involvement, with both hippocampal and amygdalar atrophy [4-6]. However, these studies have investigated the whole hippocampus or amygdala and no previous studies have looked at the

\footnotetext{
* Correspondence: j.rohrer@ucl.ac.uk

'Dementia Research Centre, Department of Neurodegenerative Disease, UCL Queen Square Institute of Neurology, University College London, 8-11 Queen Square, London WC1N 3BG, UK

Full list of author information is available at the end of the article
}

subregions of the medial temporal lobe. In this study, we therefore aimed to investigate the pattern of atrophy of the subregions of the hippocampus and the amygdala in svPPA, focusing on the involvement at different stages in order to understand the areas involved early in the disease process.

\section{Methods}

We reviewed the UCL Dementia Research Centre FTD MRI database to identify patients with a diagnosis of svPPA [7] and a usable $3 \mathrm{~T}$ T1-weighted magnetic resonance (MR) scan. Twenty-four patients were identified, all with left-temporal predominant disease. Seventy-two cognitively normal subjects with a usable volumetric $3 \mathrm{~T}$ T1-weighted MRI were identified as controls. The study

(c) The Author(s). 2019 Open Access This article is distributed under the terms of the Creative Commons Attribution 4.0 International License (http://creativecommons.org/licenses/by/4.0/), which permits unrestricted use, distribution, and 
was approved by the local ethics committee, and written informed consent was obtained from all participants. The study was conducted in accordance with the Helsinki Declaration of 1975.

Based on their scores on a test of semantic knowledge (the British Picture Vocabulary Scale, BPVS, a word-picture matching task) [8], we split the svPPA patients into three equal subgroups ( $n=8$ per group) of early (BPVS $>110 / 150)$, middle (BPVS $=55-110 / 150)$ and late disease stage (BPVS $<55 / 150$ ). Patients were negative for mutations in all FTD-related genes. Two patients received post-mortem confirmation of the underlying neuropathology, both TDP-43 type C.

All patients underwent a detailed neuropsychological examination including tests of fluid intelligence (WASI Matrices), single-word comprehension (WASI Vocabulary), naming (Graded Naming Test), reading (National Adult Reading Test), verbal memory (Recognition Memory Test for Words), visual memory (Recognition Memory Test for Faces), short-term memory (forwards digit span), working memory (backwards digit span), calculation (Graded Difficulty Calculation Test), visuoperceptual function (Visual Object and Space Perception battery Object Decision subtest) and executive function (inhibition-D-KEFS Color-Word Ink Naming Test; abstract reasoning-WASI Similarities). A percentile score based on standard norms was generated for each patient, with a mean percentile score created for the early, middle and late stage groups. Assessment of behavioural symptoms was performed using the revised version of the Cambridge Behavioural Inventory (CBI-R) [9]: six subscores were used (difficulties with self-care, abnormal sleep, hallucinations/delusions, disinhibition, abnormal eating behaviour, obsessive-compulsive behaviour, apathy and loss of empathy) with a percentage of the total possible subscore generated for every patient; for each stage, a mean percentage score was created. We report the cognitive and behavioural profiles at each stage for illustrative purposes (Fig. 1 and Additional file 1: Table S1).

T1-weighted MRIs were acquired using a 3-T scanner, either a Trio (Siemens, Erlangen, Germany, TR $=2200 \mathrm{~ms}$, $\mathrm{TI}=900 \mathrm{~ms}, \mathrm{TE}=2.9 \mathrm{~ms}$, acquisition matrix $=256 \times 256$, spatial resolution $=1.1 \mathrm{~mm}$ ) or a Prisma (Siemens, Erlangen, Germany, $\mathrm{TR}=2000 \mathrm{~ms}, \mathrm{TI}=850 \mathrm{~ms}, \mathrm{TE}=2.93$ ms, acquisition matrix $=256 \times 256$, spatial resolution $=$ $1.1 \mathrm{~mm}$ ). Individuals with moderate to severe vascular disease or space-occupying lesions were excluded.

Volumetric MRI scans were first bias field corrected and whole-brain parcellated using the geodesic information flow (GIF) algorithm [10], which is based on atlas propagation and label fusion. The hippocampal subfields and amygdalar subregions were subsequently segmented using a customized version of the module available in FreeSurfer $6.0[11,12]$, to adapt the output of GIF to the
FreeSurfer format. For the hippocampal subfields, we focused on seven areas: CA1, CA2/CA3, CA4, dentate gyrus, subiculum, presubiculum and the tail. We excluded from the analysis the hippocampus-amygdala transition area, the parasubiculum, the molecular layer of the hippocampus, the fimbria and the hippocampal fissure, as they were too small, or not reliably delineated on T1-weighted images. For the amygdalar subnuclei, we focused the analysis on five regions, by combining the smallest subnuclei, based on an anatomical subdivision [13]: lateral nucleus, basal and paralaminar nucleus, accessory basal nucleus, cortico-amygdaloid transition area and the superficial nuclei (central nucleus, cortical nucleus, medial nucleus, anterior amygdaloid area).

For comparison with the medial temporal subregions, we extracted volumes of the following cortical regions from GIF: temporal (medial, lateral, supratemporal, temporal pole), frontal (orbitofrontal, prefrontal), parietal, occipital, insular and cingulate (anterior and posterior). We also extracted volumes of subcortical structures for the pallidum, putamen, caudate, nucleus accumbens and thalamus.

Left and right volumes were corrected for total intracranial volume (TIV), computed with SPM12 v6470 (Statistical Parametric Mapping, Wellcome Trust Centre for Neuroimaging, London, UK) running under Matlab R2014b (Math Works, Natick, MA, USA) [14]. All segmentations were visually checked for quality.

Statistical analyses were performed on brain volumes (as a percentage of TIV) in STATA v14 (Stata-Corp, College Station, TX), between control and patients (early, middle and late stage groups), using a linear regression test adjusting for scanner type, TIV, gender and age. The results were corrected for multiple comparisons (Bonferroni correction): $p<0.006$ for amygdalar subnuclei and subcortical structures, $p<0.005$ for hippocampal subfields and $p<0.0035$ for cortical regions.

\section{Results}

No significant age difference was seen between any of the svPPA groups and controls [Early: 66.9 (5.5) years, Middle: 64.5 (9.5), Late: 64.2 (5.5); Controls: 61.0 (12.1)], $p=0.112, t$ test. However, there was a significant difference in gender distribution across stages [Early: $88 \%$ male, Middle: $63 \%$ male, Late: $25 \%$ male; Controls: $40 \%$ male], $p=0.032$, Chi-square test.

Amygdalar subnuclei, hippocampal subfields, cortical regions, subcortical structures, neuropsychology performance and behavioural symptoms at each stage are shown in Fig. 1.

\section{Early stage}

All the left amygdalar and hippocampal subregions (except for the tail) were affected (24-35\% and $21-27 \%$ 


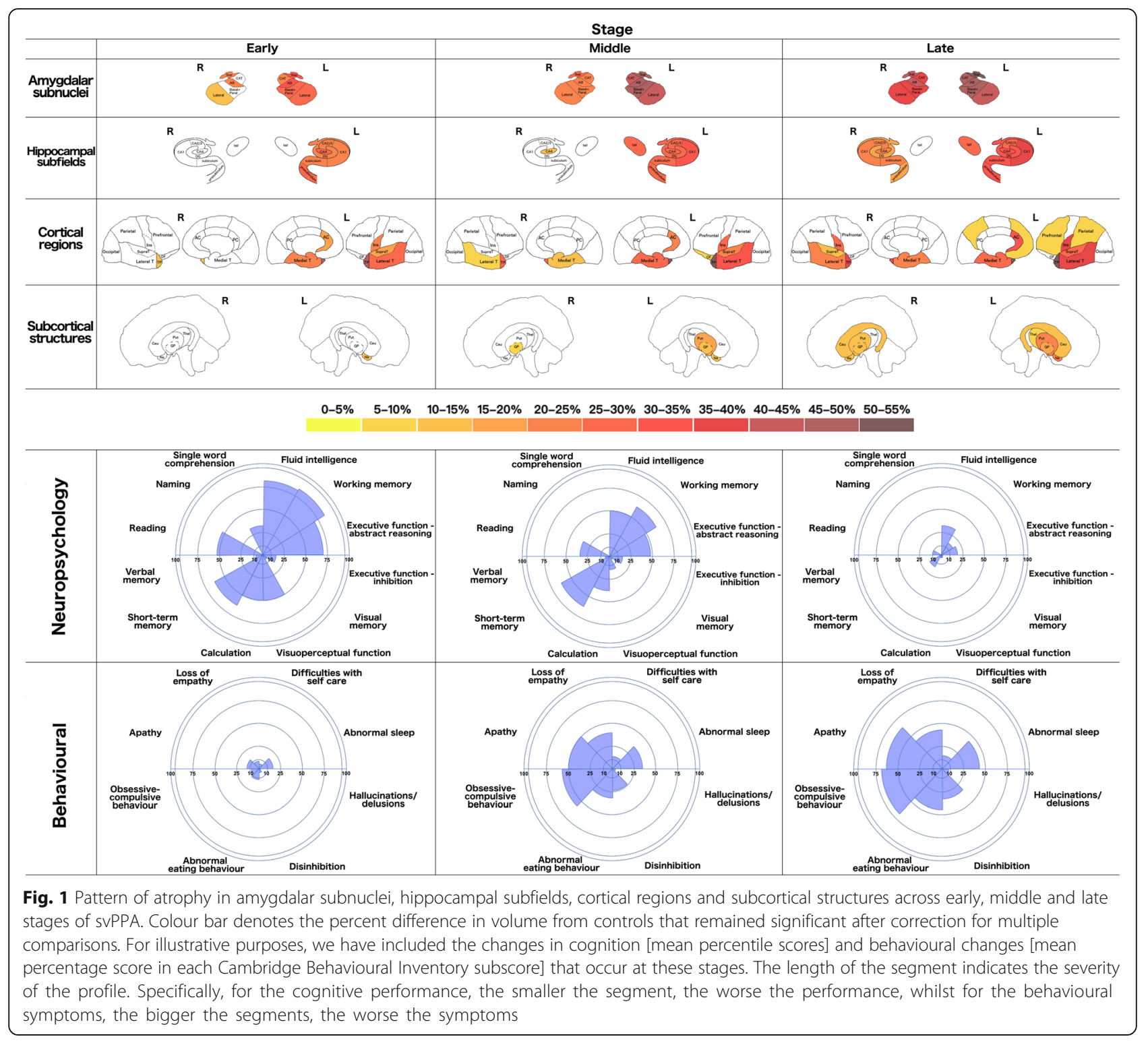

smaller than controls, $p<0.0005)$ at this stage, together with the right lateral, accessory basal and superficial nuclei of the amygdala $(15-23 \%, p<0.004)$ (Table 1$)$.

Outside of the medial temporal lobe, on the left, all the temporal cortical regions $(19-47 \%, p<0.0005)$ were affected as well as the anterior cingulate $(18 \%, p=0.001)$ and insula $(24 \%, p<0.0005)$. The left nucleus accumbens was the only other subcortical structure affected $(13 \%, p$ $<0.0005$ ). Apart from the affected amygdalar subnuclei, the only other right hemisphere structure affected at this stage was the temporal pole $(13 \%, p=0.006)$.

Cognitively, patients showed severely impaired naming already, with relatively preserved working memory, abstract reasoning and fluid intelligence. Behavioural symptoms were mild and mainly related to abnormal eating behaviour, apathy and abnormal sleep.

\section{Middle stage}

At this stage, the left hippocampal tail became affected $(28 \%, p<0.0005)$, together with the other right amygdalar nuclei $(22-26 \%, p<0.0005)$ and the right CA4 region of the hippocampus $(15 \%, p=0.003)$.

Cortically, the left orbitofrontal lobe was affected at this stage along with more posterior temporal structures on the right: lateral and medial temporal cortices (9$12 \%, p<0.0005)$. Subcortically, the left pallidum and putamen were affected $(12-17 \%, p<0.0005)$ and the right pallidum (8\%).

Cognitively, single-word comprehension and reading became increasingly impaired, but working memory, short-term memory and abstract reasoning remained relatively intact. Behavioural symptoms increased with the presence of obsessive-compulsive behaviour and loss 
Table 1 Volumetry of amygdalar subnuclei, hippocampal subfields, cortical regions and subcortical structures

\begin{tabular}{|c|c|c|c|c|c|c|c|c|c|c|c|c|c|c|c|c|}
\hline & & & \multicolumn{2}{|c|}{ Controls } & \multicolumn{2}{|c|}{ Early } & \multicolumn{2}{|c|}{ Middle } & \multirow{2}{*}{\multicolumn{2}{|c|}{ Right }} & \multicolumn{2}{|c|}{ Controls } & \multicolumn{2}{|l|}{ Early } & \multicolumn{2}{|c|}{ Middle } \\
\hline & \multicolumn{8}{|l|}{ Left } & & & & & & & & \\
\hline & Mean & SD & $\%$ & $p$-value & $\%$ & $p$-value & $\%$ & $p$-value & Mean & SD & $\%$ & $p$-value & $\%$ & $p$-value & $\%$ & $p$-value \\
\hline \multicolumn{17}{|c|}{ Amygdalar Subnuclei } \\
\hline \multicolumn{17}{|c|}{ Lateral nucleus } \\
\hline Controls & 0.045 & 0.005 & & & & & & & 0.047 & 0.004 & & & & & & \\
\hline Early & 0.033 & 0.010 & 27 & $<0.0005$ & & & & & 0.040 & 0.006 & 15 & 0.003 & & & & \\
\hline Middle & 0.026 & 0.003 & 43 & $<0.0005$ & 23 & $<0.0005$ & & & 0.035 & 0.005 & 25 & $<0.0005$ & 12 & 0.005 & & \\
\hline Late & 0.025 & 0.003 & 44 & $<0.0005$ & 24 & $<0.0005$ & 2 & 0.723 & 0.030 & 0.005 & 36 & $<0.0005$ & 25 & $<0.0005$ & 14 & 0.017 \\
\hline \multicolumn{17}{|c|}{ Basal and paralaminar nucleus } \\
\hline Controls & 0.033 & 0.004 & & & & & & & 0.034 & 0.003 & & & & & & \\
\hline Early & 0.024 & 0.006 & 29 & $<0.0005$ & & & & & 0.029 & 0.006 & 15 & 0.012 & & & & \\
\hline Middle & 0.018 & 0.003 & 46 & $<0.0005$ & 24 & $<0.0005$ & & & 0.026 & 0.004 & 22 & $<0.0005$ & 8 & 0.092 & & \\
\hline Late & 0.017 & 0.002 & 48 & $<0.0005$ & 27 & $<0.0005$ & 4 & 0.483 & 0.021 & 0.003 & 39 & $<0.0005$ & 29 & $<0.0005$ & 22 & $<0.0005$ \\
\hline \multicolumn{17}{|c|}{ Accessory basal nucleus } \\
\hline Controls & 0.018 & 0.002 & & & & & & & 0.018 & 0.002 & & & & & & \\
\hline Early & 0.012 & 0.004 & 32 & $<0.0005$ & & & & & 0.015 & 0.004 & 21 & $<0.0005$ & & & & \\
\hline Middle & 0.010 & 0.002 & 46 & $<0.0005$ & 20 & 0.002 & & & 0.014 & 0.002 & 24 & $<0.0005$ & 4 & 0.373 & & \\
\hline Late & 0.009 & 0.001 & 49 & $<0.0005$ & 25 & $<0.0005$ & 6 & 0.482 & 0.011 & 0.002 & 42 & $<0.0005$ & 27 & $<0.0005$ & 24 & 0.002 \\
\hline \multicolumn{17}{|c|}{ Cortico-amygdaloid transition area } \\
\hline Controls & 0.012 & 0.002 & & & & & & & 0.012 & 0.001 & & & & & & \\
\hline Early & 0.009 & 0.002 & 24 & $<0.0005$ & & & & & 0.011 & 0.003 & 12 & 0.157 & & & & \\
\hline Middle & 0.007 & 0.001 & 44 & $<0.0005$ & 27 & $<0.0005$ & & & 0.009 & 0.002 & 24 & $<0.0005$ & 14 & 0.025 & & \\
\hline Late & 0.006 & 0.001 & 48 & $<0.0005$ & 32 & $<0.0005$ & 7 & 0.339 & 0.008 & 0.002 & 36 & $<0.0005$ & 28 & $<0.0005$ & 16 & 0.049 \\
\hline \multicolumn{17}{|c|}{ Superficial nuclei (Ce, Co, Me, AAA) } \\
\hline Controls & 0.011 & 0.002 & & & & & & & 0.012 & 0.002 & & & & & & \\
\hline Early & 0.007 & 0.002 & 35 & $<0.0005$ & & & & & 0.009 & 0.002 & 23 & 0.004 & & & & \\
\hline Middle & 0.006 & 0.001 & 47 & $<0.0005$ & 18 & 0.005 & & & 0.009 & 0.001 & 26 & $<0.0005$ & 4 & 0.341 & & \\
\hline Late & 0.005 & 0.001 & 51 & $<0.0005$ & 25 & $<0.0005$ & 9 & 0.275 & 0.007 & 0.002 & 41 & $<0.0005$ & 24 & 0.002 & 21 & 0.024 \\
\hline \multicolumn{17}{|c|}{ Hippocampal Subfields } \\
\hline \multicolumn{17}{|l|}{ CA1 } \\
\hline Controls & 0.044 & 0.005 & & & & & & & 0.047 & 0.006 & & & & & & \\
\hline Early & 0.035 & 0.005 & 22 & $<0.0005$ & & & & & 0.045 & 0.008 & 5 & 0.995 & & & & \\
\hline Middle & 0.031 & 0.007 & 31 & $<0.0005$ & 11 & 0.020 & & & 0.043 & 0.007 & 8 & 0.138 & 3 & 0.267 & & \\
\hline Late & 0.029 & 0.004 & 36 & $<0.0005$ & 18 & 0.001 & 7 & 0.268 & 0.036 & 0.006 & 24 & $<0.0005$ & 19 & $<0.0005$ & 17 & 0.003 \\
\hline \multicolumn{17}{|l|}{$\mathrm{CA} 2 / \mathrm{CA} 3$} \\
\hline Controls & 0.016 & 0.002 & & & & & & & 0.017 & 0.002 & & & & & & \\
\hline Early & 0.012 & 0.002 & 24 & $<0.0005$ & & & & & 0.016 & 0.004 & 6 & 0.931 & & & & \\
\hline Middle & 0.011 & 0.002 & 27 & $<0.0005$ & 3 & 0.460 & & & 0.015 & 0.003 & 12 & 0.064 & 7 & 0.184 & & \\
\hline Late & 0.012 & 0.002 & 26 & $<0.0005$ & 2 & 0.518 & -1 & 0.945 & 0.013 & 0.002 & 24 & $<0.0005$ & 19 & 0.002 & 13 & 0.054 \\
\hline \multicolumn{17}{|l|}{ CA4 } \\
\hline Controls & 0.018 & 0.002 & & & & & & & 0.019 & 0.002 & & & & & & \\
\hline Early & 0.013 & 0.002 & 27 & $<0.0005$ & & & & & 0.017 & 0.004 & 10 & 0.281 & & & & \\
\hline Middle & 0.013 & 0.001 & 27 & $<0.0005$ & 1 & 0.342 & & & 0.016 & 0.002 & 15 & 0.003 & 5 & 0.156 & & \\
\hline Late & 0.012 & 0.001 & 34 & $<0.0005$ & 9 & 0.007 & 9 & 0.066 & 0.015 & 0.002 & 21 & $<0.0005$ & 13 & 0.004 & 8 & 0.111 \\
\hline
\end{tabular}


Table 1 Volumetry of amygdalar subnuclei, hippocampal subfields, cortical regions and subcortical structures (Continued)

\begin{tabular}{|c|c|c|c|c|c|c|c|c|c|c|c|c|c|c|c|c|}
\hline & \multirow{2}{*}{\multicolumn{2}{|c|}{ Left }} & \multicolumn{2}{|c|}{ Controls } & \multicolumn{2}{|c|}{ Early } & \multicolumn{2}{|c|}{ Middle } & \multirow{2}{*}{\multicolumn{2}{|c|}{ Right }} & \multicolumn{2}{|c|}{ Controls } & \multicolumn{2}{|l|}{ Early } & \multicolumn{2}{|c|}{ Middle } \\
\hline & & & & & & & & & & & & & & & & \\
\hline & Mean & SD & $\%$ & $p$-value & $\%$ & $p$-value & $\%$ & $p$-value & Mean & SD & $\%$ & $p$-value & $\%$ & $p$-value & $\%$ & $p$-value \\
\hline \multicolumn{17}{|c|}{ Dentate gyrus } \\
\hline Controls & 0.021 & 0.002 & & & & & & & 0.021 & 0.002 & & & & & & \\
\hline Early & 0.016 & 0.002 & 25 & $<0.0005$ & & & & & 0.020 & 0.005 & 7 & 0.759 & & & & \\
\hline Middle & 0.015 & 0.002 & 27 & $<0.0005$ & 4 & 0.183 & & & 0.019 & 0.003 & 13 & 0.021 & 6 & 0.132 & & \\
\hline Late & 0.014 & 0.002 & 32 & $<0.0005$ & 10 & 0.011 & 6 & 0.185 & 0.017 & 0.003 & 19 & $<0.0005$ & 14 & 0.003 & 8 & 0.117 \\
\hline \multicolumn{17}{|l|}{ Subiculum } \\
\hline Controls & 0.028 & 0.003 & & & & & & & 0.029 & 0.003 & & & & & & \\
\hline Early & 0.022 & 0.002 & 21 & $<0.0005$ & & & & & 0.028 & 0.006 & 1 & 0.425 & & & & \\
\hline Middle & 0.020 & 0.003 & 28 & $<0.0005$ & 10 & 0.048 & & & 0.026 & 0.005 & 8 & 0.116 & 8 & 0.074 & & \\
\hline Late & 0.020 & 0.004 & 31 & $<0.0005$ & 13 & 0.005 & 4 & 0.338 & 0.022 & 0.005 & 23 & $<0.0005$ & 23 & $<0.0005$ & 16 & 0.004 \\
\hline \multicolumn{17}{|c|}{ Presubiculum } \\
\hline Controls & 0.023 & 0.003 & & & & & & & 0.022 & 0.003 & & & & & & \\
\hline Early & 0.017 & 0.002 & 27 & $<0.0005$ & & & & & 0.023 & 0.006 & -2 & 0.173 & & & & \\
\hline Middle & 0.016 & 0.002 & 30 & $<0.0005$ & 5 & 0.362 & & & 0.021 & 0.007 & 5 & 0.942 & 6 & 0.267 & & \\
\hline Late & 0.016 & 0.003 & 33 & $<0.0005$ & 8 & 0.045 & 3 & 0.245 & 0.018 & 0.005 & 19 & 0.001 & 20 & 0.001 & 15 & 0.015 \\
\hline \multicolumn{17}{|c|}{ Hippocampal tail } \\
\hline Controls & 0.041 & 0.005 & & & & & & & 0.041 & 0.005 & & & & & & \\
\hline Early & 0.034 & 0.006 & 18 & 0.019 & & & & & 0.043 & 0.010 & -4 & 0.055 & & & & \\
\hline Middle & 0.030 & 0.005 & 28 & $<0.0005$ & 12 & 0.026 & & & 0.042 & 0.010 & -2 & 0.371 & 2 & 0.41 & & \\
\hline Late & 0.029 & 0.006 & 29 & $<0.0005$ & 13 & 0.009 & 2 & 0.624 & 0.037 & 0.008 & 8 & 0.084 & 12 & 0.008 & 10 & 0.054 \\
\hline \multicolumn{17}{|c|}{ Cortical Regions } \\
\hline \multicolumn{17}{|l|}{ Orbitofrontal } \\
\hline Controls & 0.697 & 0.047 & & & & & & & 0.716 & 0.048 & & & & & & \\
\hline Early & 0.682 & 0.045 & 2 & 0.934 & & & & & 0.727 & 0.057 & -2 & 0.158 & & & & \\
\hline Middle & 0.629 & 0.089 & 10 & 0.001 & 8 & 0.015 & & & 0.716 & 0.046 & 0 & 0.806 & 1 & 0.362 & & \\
\hline Late & 0.637 & 0.063 & 9 & 0.009 & 7 & 0.062 & -1 & 0.612 & 0.697 & 0.078 & 3 & 0.647 & 4 & 0.166 & 3 & 0.604 \\
\hline \multicolumn{17}{|c|}{ Prefrontal cortex } \\
\hline Controls & 4.216 & 0.230 & & & & & & & 4.322 & 0.224 & & & & & & \\
\hline Early & 4.087 & 0.337 & 3 & 0.691 & & & & & 4.299 & 0.379 & 1 & 0.545 & & & & \\
\hline Middle & 4.045 & 0.529 & 4 & 0.112 & 1 & 0.373 & & & 4.380 & 0.369 & -1 & 0.506 & -2 & 0.977 & & \\
\hline Late & 3.806 & 0.250 & 10 & 0.002 & 7 & 0.047 & 6 & 0.245 & 4.119 & 0.269 & 5 & 0.201 & 4 & 0.168 & 6 & 0.153 \\
\hline \multicolumn{17}{|c|}{ Anterior cingulate } \\
\hline Controls & 0.382 & 0.039 & & & & & & & 0.283 & 0.042 & & & & & & \\
\hline Early & 0.315 & 0.041 & 18 & 0.001 & & & & & 0.289 & 0.046 & -2 & 0.339 & & & & \\
\hline Middle & 0.300 & 0.068 & 22 & $<0.0005$ & 5 & 0.311 & & & 0.318 & 0.069 & -13 & 0.008 & -10 & 0.204 & & \\
\hline Late & 0.255 & 0.026 & 33 & $<0.0005$ & 19 & 0.002 & 15 & 0.023 & 0.288 & 0.058 & -2 & 0.968 & 0 & 0.457 & 9 & 0.047 \\
\hline \multicolumn{17}{|c|}{ Posterior cingulate } \\
\hline Controls & 0.359 & 0.038 & & & & & & & 0.343 & 0.035 & & & & & & \\
\hline Early & 0.350 & 0.020 & 3 & 0.609 & & & & & 0.368 & 0.019 & -7 & 0.009 & & & & \\
\hline Middle & 0.332 & 0.025 & 7 & 0.065 & 5 & 0.320 & & & 0.365 & 0.028 & -6 & 0.022 & 1 & 0.747 & & \\
\hline Late & 0.337 & 0.028 & 6 & 0.169 & 4 & 0.535 & -1 & 0.728 & 0.361 & 0.047 & -5 & 0.150 & 2 & 0.348 & 1 & 0.523 \\
\hline
\end{tabular}


Table 1 Volumetry of amygdalar subnuclei, hippocampal subfields, cortical regions and subcortical structures (Continued)

\begin{tabular}{|c|c|c|c|c|c|c|c|c|c|c|c|c|c|c|c|c|}
\hline & & & \multicolumn{2}{|c|}{ Controls } & \multicolumn{2}{|c|}{ Early } & \multicolumn{2}{|c|}{ Middle } & \multirow{2}{*}{\multicolumn{2}{|c|}{ Right }} & \multicolumn{2}{|c|}{ Controls } & \multicolumn{2}{|l|}{ Early } & \multicolumn{2}{|c|}{ Middle } \\
\hline & \multicolumn{8}{|l|}{ Left } & & & & & & & & \\
\hline & Mean & SD & $\%$ & $p$-value & $\%$ & $p$-value & $\%$ & $p$-value & Mean & SD & $\%$ & $p$-value & $\%$ & $p$-value & $\%$ & $p$-value \\
\hline \multicolumn{17}{|l|}{ Parietal } \\
\hline Controls & 3.224 & 0.211 & & & & & & & 3.186 & 0.229 & & & & & & \\
\hline Early & 3.143 & 0.229 & 3 & 0.538 & & & & & 3.216 & 0.248 & -1 & 0.049 & & & & \\
\hline Middle & 3.147 & 0.249 & 2 & 0.709 & 0 & 0.450 & & & 3.272 & 0.200 & -3 & 0.053 & -2 & 0.944 & & \\
\hline Late & 2.993 & 0.234 & 7 & 0.003 & 5 & 0.008 & 5 & 0.046 & 3.142 & 0.213 & 1 & 0.793 & 2 & 0.096 & 4 & 0.105 \\
\hline \multicolumn{17}{|l|}{ Occipital } \\
\hline Controls & 2.473 & 0.207 & & & & & & & 2.564 & 0.205 & & & & & & \\
\hline Early & 2.393 & 0.227 & 3 & 0.835 & & & & & 2.538 & 0.195 & 1 & 0.575 & & & & \\
\hline Middle & 2.395 & 0.155 & 3 & 0.552 & 0 & 0.776 & & & 2.552 & 0.175 & 0 & 0.697 & -1 & 0.887 & & \\
\hline Late & 2.432 & 0.148 & 2 & 0.733 & -2 & 0.926 & -2 & 0.853 & 2.572 & 0.147 & 0 & 0.796 & -1 & 0.817 & -1 & 0.924 \\
\hline \multicolumn{17}{|l|}{ Insula } \\
\hline Controls & 0.370 & 0.035 & & & & & & & 0.381 & 0.039 & & & & & & \\
\hline Early & 0.281 & 0.032 & 24 & $<0.0005$ & & & & & 0.343 & 0.049 & 10 & 0.110 & & & & \\
\hline Middle & 0.260 & 0.036 & 30 & $<0.0005$ & 7 & 0.064 & & & 0.337 & 0.038 & 12 & 0.007 & 2 & 0.425 & & \\
\hline Late & 0.229 & 0.021 & 38 & $<0.0005$ & 18 & $<0.0005$ & 12 & 0.013 & 0.267 & 0.039 & 30 & $<0.0005$ & 22 & $<0.0005$ & 21 & $<0.0005$ \\
\hline \multicolumn{17}{|c|}{ Medial temporal } \\
\hline Controls & 1.012 & 0.062 & & & & & & & 1.041 & 0.067 & & & & & & \\
\hline Early & 0.785 & 0.057 & 22 & $<0.0005$ & & & & & 0.981 & 0.070 & 6 & 0.076 & & & & \\
\hline Middle & 0.730 & 0.056 & 28 & $<0.0005$ & 7 & 0.042 & & & 0.915 & 0.070 & 12 & $<0.0005$ & 7 & 0.044 & & \\
\hline Late & 0.743 & 0.058 & 27 & $<0.0005$ & 5 & 0.088 & -2 & 0.787 & 0.791 & 0.074 & 24 & $<0.0005$ & 19 & $<0.0005$ & 14 & $<0.0005$ \\
\hline \multicolumn{17}{|c|}{ Lateral temporal } \\
\hline Controls & 2.304 & 0.153 & & & & & & & 2.345 & 0.143 & & & & & & \\
\hline Early & 1.652 & 0.201 & 28 & $<0.0005$ & & & & & 2.231 & 0.134 & 5 & 0.133 & & & & \\
\hline Middle & 1.554 & 0.150 & 33 & $<0.0005$ & 6 & 0.084 & & & 2.137 & 0.099 & 9 & $<0.0005$ & 4 & 0.105 & & \\
\hline Late & 1.384 & 0.159 & 40 & $<0.0005$ & 16 & $<0.0005$ & 11 & 0.026 & 1.864 & 0.217 & 21 & $<0.0005$ & 16 & $<0.0005$ & 13 & $<0.0005$ \\
\hline \multicolumn{17}{|c|}{ Temporal pole } \\
\hline Controls & 0.488 & 0.056 & & & & & & & 0.477 & 0.055 & & & & & & \\
\hline Early & 0.261 & 0.066 & 47 & $<0.0005$ & & & & & 0.413 & 0.071 & 13 & 0.006 & & & & \\
\hline Middle & 0.231 & 0.035 & 53 & $<0.0005$ & 12 & 0.187 & & & 0.352 & 0.049 & 26 & $<0.0005$ & 15 & 0.019 & & \\
\hline Late & 0.228 & 0.029 & 53 & $<0.0005$ & 13 & 0.324 & 1 & 0.766 & 0.287 & 0.038 & 40 & $<0.0005$ & 30 & $<0.0005$ & 18 & 0.048 \\
\hline \multicolumn{17}{|c|}{ Supratemporal } \\
\hline Controls & 0.430 & 0.050 & & & & & & & 0.369 & 0.039 & & & & & & \\
\hline Early & 0.348 & 0.037 & 19 & $<0.0005$ & & & & & 0.357 & 0.045 & 3 & 0.910 & & & & \\
\hline Middle & 0.336 & 0.046 & 22 & $<0.0005$ & 4 & 0.359 & & & 0.368 & 0.040 & 0 & 0.718 & -3 & 0.855 & & \\
\hline Late & 0.301 & 0.056 & 30 & $<0.0005$ & 14 & 0.017 & 10 & 0.122 & 0.322 & 0.054 & 13 & 0.004 & 10 & 0.028 & 12 & 0.016 \\
\hline \multicolumn{17}{|c|}{ Subcortical Structures } \\
\hline \multicolumn{17}{|c|}{ Nucleus accumbens } \\
\hline Controls & 0.040 & 0.003 & & & & & & & 0.038 & 0.003 & & & & & & \\
\hline Early & 0.035 & 0.003 & 13 & $<0.0005$ & & & & & 0.035 & 0.003 & 9 & 0.048 & & & & \\
\hline Middle & 0.034 & 0.005 & 15 & $<0.0005$ & 3 & 0.235 & & & 0.036 & 0.004 & 5 & 0.155 & -4 & 0.638 & & \\
\hline Late & 0.030 & 0.003 & 24 & $<0.0005$ & 13 & 0.001 & 10 & 0.019 & 0.032 & 0.004 & 15 & $<0.0005$ & 7 & 0.026 & 11 & 0.007 \\
\hline
\end{tabular}


Table 1 Volumetry of amygdalar subnuclei, hippocampal subfields, cortical regions and subcortical structures (Continued)

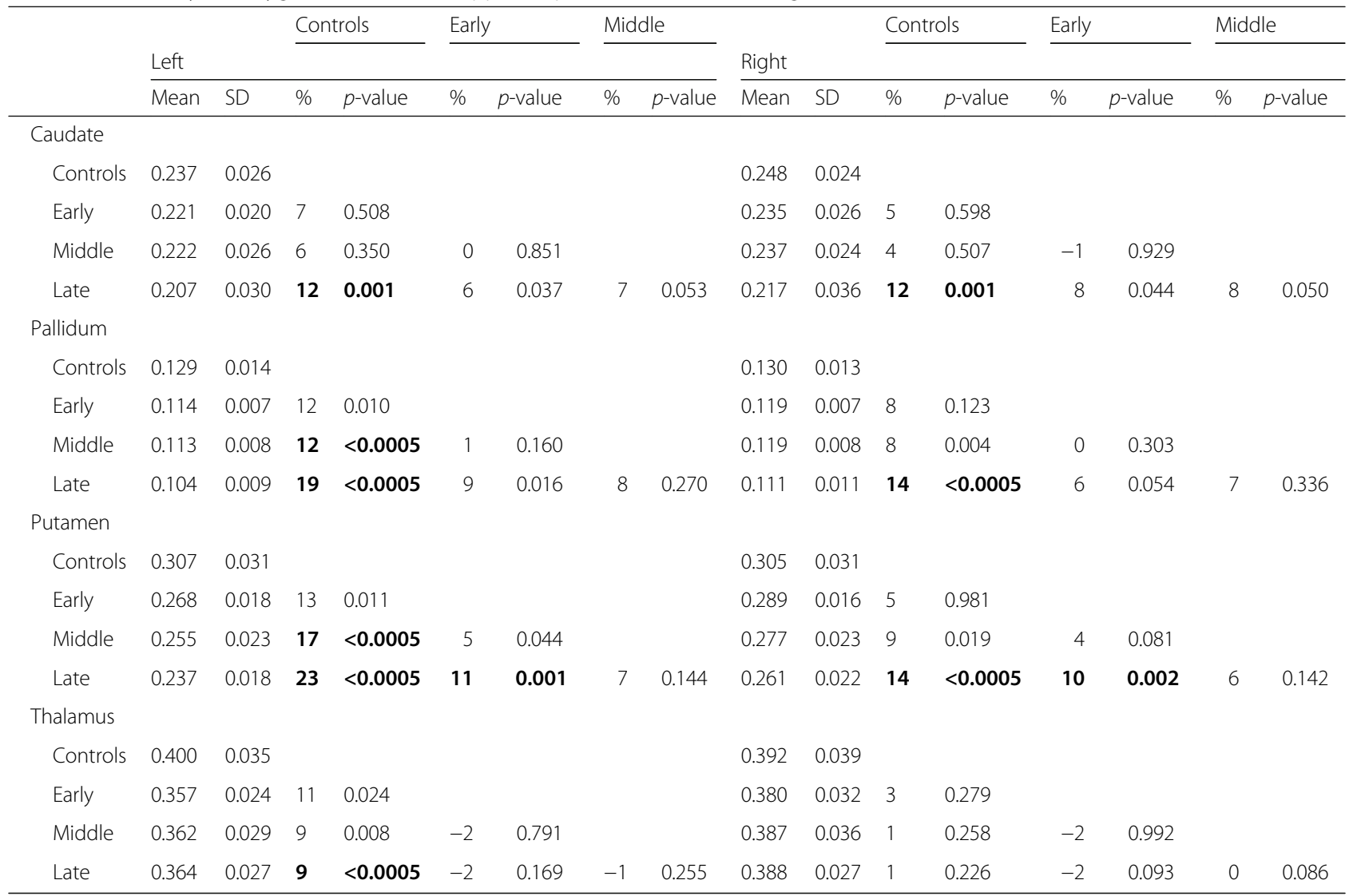

Values denote mean and standard deviation (SD) volumes as the percentage of the total intracranial volume (TIV) or difference (\%). $p$ values denote significance on linear regression test. Bold represents a significant difference between the groups after correcting for multiple comparisons

of empathy as well as abnormal eating behaviour, apathy and disinhibition.

\section{Late stage}

In the late stage, the remaining right hippocampal regions (except the tail) $(19-24 \%, p<0.001)$ became affected.

Cortically, spread to the left prefrontal and parietal cortices was seen whilst on the right, the insula (30\%) and supratemporal cortex $(13 \%, p<0.004)$ were affected. Subcortically, the left caudate, thalamus and right nucleus accumbens, caudate and putamen were affected (12-15\%).

At this stage, all cognitive domains were severely impaired except for short-term and working memory, abstract reasoning and fluid intelligence. Severe behavioural symptoms were seen.

\section{Discussion}

Using advanced subregional segmentation, we were able to detect early involvement in the right hemisphere in svPPA, with progression of atrophy through the medial temporal lobes as the disease moves from early to middle to late stage.
Extensive medial temporal atrophy is seen on the left in most amygdalar and hippocampal subregions at the earliest stage of svPPA, co-incidental with the involvement of all of the temporal cortices on the left. This is consistent with previous studies showing that even at first clinical presentation, significant left temporal lobe atrophy is present $[1,15]$.

Previous studies have not shown early involvement of the right medial temporal structures. In this study, the earliest subnuclei affected on the right were the accessory basal, lateral and superficial nuclei of the amygdala. These subnuclei are interconnected and receive input from the temporal pole and the hippocampus (also affected on the right in the early stage) as well as other parts of the temporal and frontal cortices and the nucleus accumbens [13, 16]. The ability to use advanced subregional segmentation techniques in this study allows early detection of right medial temporal atrophy.

The cognitive and behavioural correlates of the individual right amygdalar subnuclei are poorly studied, but prior studies of the whole amygdala implicate the right side as being important in the processing of emotional information $[17,18]$. In our 
study, loss of empathy is mildly affected at the earliest stage (Fig. 1): this is likely to represent an impairment of self-knowledge, a process that requires the linking of emotions with semantics, and has previously been shown to be associated with right temporal lobe atrophy including the amygdala [19]. The particular amygdalar subnuclei affected early are part of the limbic network and therefore likely to be intrinsically involved in emotion processing [16].

Of all the medial temporal subregions, the hippocampal tail is preserved until the later stages of svPPA. This is in line with previous studies, where the posterior temporal lobe is spared and an antero-posterior gradient is present $[20,21]$. Indeed, svPPA patients typically show intact episodic memory and spatial navigation, functions typically linked to the hippocampal tail. Consistent with the theory of svPPA as a network-opathy [22], the first hippocampal region to become affected on the right is CA4, an area highly connected to the temporal cortex and amygdala [23].

Limitations of the study include using cross-sectional data with staging of the disease by impairment on a task of semantic knowledge and the small number of svPPA cases. Further studies would benefit from the analysis of longitudinal data from a larger sample to see whether the same pattern is seen. Despite the gold standard still being manual segmentation of dedicated MRIs or on brain tissue post-mortem, these automated methods included in this study have been previously validated and proven reliable to delineate the subregions on T1-MRI (Dice coefficients > 0.86; ICC 0.88-0.93) [10-12, 24, 25]. Moreover, in this study, we carefully excluded small subregions and combined together groups of nuclei to improve the anatomical validity. Automated segmentations will play a key role in the future, as manual segmentations are likely to be unfeasible for large cohorts of patients.

\section{Additional file}

Additional file 1: Table S1. Cognitive and behavioural variables for the svPPA patients. $p$ values denote significance on Kruskal-Wallis test among the three groups. (DOCX $17 \mathrm{~kb}$ )

\section{Acknowledgements}

Not applicable.

\section{Funding}

The Dementia Research Centre is supported by Alzheimer's Research UK, Brain Research Trust and The Wolfson Foundation. This work was supported by the NIHR Queen Square Dementia Biomedical Research Unit and the NIHR UCL/H Biomedical Research Centre, the MRC UK GENFI grant (MR/ M023664/1) and the Alzheimer's Society (AS-PG-16-007). JDR is supported by an MRC Clinician Scientist Fellowship (MR/M008525/1) and has received funding from the NIHR Rare Disease Translational Research Collaboration (BRC149/NS/MH). JDW was supported by a Wellcome Trust Senior Clinical Fellowship (091673/Z/10/Z), and his research is supported by the Alzheimer's
Society, Alzheimer's Research UK and the NIHR UCLH Biomedical Research Centre. SO is funded by the Engineering and Physical Sciences Research Council (EP/H046410/1, EP/J020990/1, EP/K005278), the Medical Research Council (MR/J01107X/1), the EU-FP7 project VPH-DARE@IT (FP7- ICT-2011-9601055) and the National Institute for Health Research University College London Hospitals Biomedical Research Centre (NIHR BRC UCLH/UCL High Impact Initiative BW.mn.BRC10269). JEl is supported by the European Research Council (Starting Grant 677697, project BUNGEE-TOOLS). MAS acknowledges the financial support by the EPSRC-funded UCL Centre for Doctoral Training in Medical Imaging (EP/L016478/1).

\section{Availability of data and materials}

The datasets used and analysed during the current study are available from the corresponding author on reasonable request.

\section{Authors' contributions}

$\mathrm{MB}$ drafted the body of the manuscript, tables and figures and ran the analyses. JDR contributed to the design and concept of the study. JEI contributed to the data analyses. LLR, CVG, CRM, JDW and JDR were responsible for the collection of data and recruitment of patients. All authors critically reviewed and approved the final manuscript and contributed to the data interpretation.

\section{Ethics approval and consent to participate}

This study was approved by the London Queen Square NRES Committee. Written informed consent was obtained from all participants.

\section{Consent for publication}

Not applicable.

\section{Competing interests}

JDR has been on a Medical Advisory Board for Wave Life Sciences and lonis Pharmaceuticals. All other authors declare that they have no competing interests.

\section{Publisher's Note}

Springer Nature remains neutral with regard to jurisdictional claims in published maps and institutional affiliations.

\section{Author details}

${ }^{1}$ Dementia Research Centre, Department of Neurodegenerative Disease, UCL Queen Square Institute of Neurology, University College London, 8-11 Queen Square, London WC1N 3BG, UK. ${ }^{2}$ Centre for Medical Image Computing, Department of Medical Physics and Biomedical Engineering, University College London, London, UK. ${ }^{3}$ School of Biomedical Engineering and Imaging Sciences, St Thomas' Hospital, King's College London, London, UK.

Received: 3 January 2019 Accepted: 2 April 2019

Published online: 10 May 2019

References

1. Rohrer JD, Warren JD, Modat M, Ridgway GR, Douiri A, Rossor MN, Ourselin S, Fox NC. Patterns of cortical thinning in the language variants of frontotemporal lobar degeneration. Neurology. 2009;72(18):1562-9.

2. Rohrer JD, Rosen HJ. Neuroimaging in frontotemporal dementia. Int Rev Psychiatry. 2013;25(2):221-9.

3. Schroeter ML, Raczka K, Neumann J, Yves von Cramon D. Towards a nosology for frontotemporal lobar degenerations-a meta-analysis involving 267 subjects. Neuroimage. 2007;36(3):497-510.

4. Rohrer JD, McNaught E, Foster J, Clegg SL, Barnes J, Omar R, Warrington EK, Rossor MN, Warren JD, Fox NC. Tracking progression in frontotemporal lobar degeneration: serial MRI in semantic dementia. Neurology. 2008; 71(18):1445-51.

5. Lehmann M, Douiri A, Kim LG, Modat M, Chan D, Ourselin S, Barnes J, Fox NC. Atrophy patterns in Alzheimer's disease and semantic dementia: a comparison of FreeSurfer and manual volumetric measurements. Neuroimage. 2010;49(3):2264-74.

6. Nestor PJ, Fryer TD, Hodges JR. Declarative memory impairments in Alzheimer's disease and semantic dementia. Neuroimage. 2006;30(3): 1010-20. 
7. Gorno-Tempini ML, Hillis AE, Weintraub S, Kertesz A, Mendez M, Cappa SF, Ogar JM, Rohrer JD, Black S, Boeve BF, Manes F, Dronkers NF, Vandenberghe R, Rascovsky K, Patterson K, Miller BL, Knopman DS, Hodges $J R$, Mesulam MM, Grossman M. Classification of primary progressive aphasia and its variants. Neurology. 2011;76(11):1006-14.

8. Dunn DM, Dunn LM, National Foundation for Educational Research in England and Wales, GL Assessment (Firm). 2009. 3rd ed. GL Assessment. ISBN-10: 0708719554

9. Wear HJ, Wedderburn CJ, Mioshi E, Williams-Gray CH, Mason SL, Barker RA, Hodges JR. The Cambridge Behavioural Inventory revised. Dement Neuropsychol. 2008;2(2):102-7.

10. Cardoso MJ, Modat M, Wolz R, Melbourne A, Cash D, Rueckert D, Ourselin S. Geodesic information flows: spatially-variant graphs and their application to segmentation and fusion. IEEE TMI. 2015. https://doi.org/10.1109/TMI.2015. 2418298.

11. Saygin ZM, Kliemann D, Iglesias JE, van der Kouwe AJW, Boyd E, Reuter M, Stevens A, Van Leemput K, McKee A, Frosch MP, Fischl B, Augustinack JC, Alzheimer's Disease Neuroimaging Initiative. High-resolution magnetic resonance imaging reveals nuclei of the human amygdala: manual segmentation to automatic atlas. Neuroimage. 2017;155:370-82.

12. Iglesias JE, Augustinack JC, Nguyen K, Player CM, Player A, Wright M, Roy N, Frosch MP, McKee AC, Wald LL, Fischl B, Van Leemput K. A computational atlas of the hippocampal formation using ex vivo, ultra-high resolution MRI: application to adaptive segmentation of in vivo MRI. Neuroimage. 2015;115: 117-37.

13. deCampo DM, Fudge JL. Where and what is the paralaminar nucleus? A review on a unique and frequently overlooked area of the primate amygdala. Neurosci Biobehav Rev. 2012;36(1):520-35.

14. Malone IB, Leung KK, Clegg S, Barnes J, Whitwell JL, Ashburner J, Fox NC, Ridgway GR. Accurate automatic estimation of total intracranial volume: a nuisance variable with less nuisance. Neuroimage. 2015;104:366-72.

15. Czarnecki K, Duffy JR, Nehl CR, Cross SA, Molano JR, Jack CR Jr, Shiung MM, Josephs KA, Boeve BF. Very early semantic dementia with progressive temporal lobe atrophy: an 8-year longitudinal study. Arch Neurol. 2008; 65(12):1659-63.

16. LeDoux J. The amygdala. Curr Biol. 2007;17(20):R868-74.

17. Rosen HJ, Perry RJ, Murphy J, Kramer JH, Mychack P, Schuff N, Weiner M, Levenson RW, Miller BL. Emotion comprehension in the temporal variant of frontotemporal dementia. Brain. 2002;125(Pt 10):2286-95.

18. Snowden JS, Harris JM, Thompson JC, Kobylecki C, Jones M, Richardson AM, Neary D. Semantic dementia and the left and right temporal lobes. Cortex. 2017. https://doi.org/10.1016/j.cortex.2017.08.024.

19. Sollberger M, Rosen HJ, Shany-Ur T, Ullah J, Stanley CM, Laluz V, Weiner MW, Wilson SM, Miller BL, Rankin KP. Neural substrates of socioemotional self-awareness in neurodegenerative disease. Brain Behav. 2014;4(2):201-14.

20. La Joie R, Perrotin A, de La Sayette V, Egret S, Doeuvre L, Belliard S, Eustache F, Desgranges B, Chételat G. Hippocampal subfield volumetry in mild cognitive impairment, Alzheimer's disease and semantic dementia. Neuroimage Clin. 2013;3:155-62.

21. Tan RH, Wong S, Kril JJ, Piquet O, Hornberger M, Hodges JR, Halliday GM. Beyond the temporal pole: limbic memory circuit in the semantic variant of primary progressive aphasia. Brain. 2014;137(Pt 7:2065-76.

22. Fletcher PD, Warren JD. Semantic dementia: a specific network-opathy. J Mol Neurosci. 2011;45(3):629-36.

23. de Flores R, Mutlu J, Bejanin A, Gonneaud J, Landeau B, Tomadesso C, Mézenge F, de La Sayette V, Eustache F, Chételat G. Intrinsic connectivity of hippocampal subfields in normal elderly and mild cognitive impairment patients. Hum Brain Mapp. 2017;38(10):4922-32.

24. Herten A, Konrad K, Krinzinger H, Seitz J, von Polier GG. Accuracy and bias of automatic hippocampal segmentation in children and adolescents. Brain Struct Funct. 2018. https://doi.org/10.1007/s00429-018-1802-2.

25. Whelan CD, Hibar DP, van Velzen LS, Zannas AS, Carrillo-Roa T, McMahon K, Prasad G, Kelly S, Faskowitz J, deZubiracay G, Iglesias JE, van Erp TGM, Frodl T, Martin NG, Wright MJ, Jahanshad N, Schmaal L, Sämann PG, Thompson PM, Alzheimer's Disease Neuroimaging Initiative. Heritability and reliability of automatically segmented human hippocampal formation subregions. Neuroimage. 2016;128:125-37.

\section{Ready to submit your research? Choose BMC and benefit from:}

- fast, convenient online submission

- thorough peer review by experienced researchers in your field

- rapid publication on acceptance

- support for research data, including large and complex data types

- gold Open Access which fosters wider collaboration and increased citations

- maximum visibility for your research: over $100 \mathrm{M}$ website views per year

At BMC, research is always in progress.

Learn more biomedcentral.com/submissions 